\title{
Association between Ki67 Index and Clinicopatho- logical Features in Colorectal Cancer
}

\author{
Pan Li $^{\mathrm{a}}$ Zhi-Tao Xiao ${ }^{\mathrm{b}}$ Todd A. Braciak ${ }^{\mathrm{a}}$ Qing-Jan Ou ${ }^{\mathrm{b}}$ Gong Chen ${ }^{\mathrm{b}}$ Fuat S. Oduncu ${ }^{\mathrm{a}}$ \\ a Department of Hematology and Oncology, Medizinische Klinik und Poliklinik IV, Klinikum der Universität München, Munich, Germany; \\ ${ }^{b}$ Department of Colorectal Surgery, State Key Laboratory of Oncology in South China, Sun Yat-sen University Cancer Center, Guangzhou, \\ China
}

Keywords

Colorectal cancer · Ki67 · Prognosis · Biomarker

\section{Summary}

Background: Conflicting results have been reported about the association between the Ki67 labeling index (Ki67-Li) and clinical outcome in patients with colorectal cancer (CRC). Patients and Methods: Ki67 expression was assessed by immunohistochemistry (IHC) in 2,233 consecutive CRC cases. Results: We determined 992 cases to have a low and 1,241 cases to have a high Ki67Li (representing an approximately 44-56\% breakdown in distribution between low versus high patients designated by phenotype). Stage III patients with a high Ki67Li had higher 3-year disease-free survival (DFS) and overall survival (OS) than those with a low Ki67-Li (DFS 70 vs. $61 \%$; $p=0.02$ and OS 75 vs. $64 \% ; p=0.008$ ). We also found significantly improved 3-year progressionfree survival (PFS) for stage IV patients in the high versus the low Ki67-Li group (PFS 14 vs. 10\%; p = 0.02). Yet, we found no statistical differences in prognosis for stage I and II patients and in OS for stage IV patients between high versus low Ki67-Li ( $p>0.05$ ). Conclusion: Our results suggest that high Ki67-Li can be an independent prognostic biomarker to aid the assessment of patient outcomes in both stage III and IV CRC.

(C) 2016 S. Karger GmbH, Freiburg

Correspondence can also be sent to: Dr. Gong Chen, Department of Colorectal Surgery, State Key Laboratory of Oncology in South China, Sun Yat-sen University Cancer Center, East Dongfeng Road 651, 510060 Guangzhou, China, e-mail: chengong@sysucc.org.cn

\section{Introduction}

Colorectal cancer (CRC) is the third most common cancer in men and the second most common cancer in women worldwide [1]. Data collected in 2008 showed that worldwide there were over 1.23 million new cases, and over 608,000 patients died from CRC that year [2]. Several factors influence the prognosis, including clinical, histopathological, and biological factors related to the stage of the CRC tumor. Therefore, investigations into the molecular mechanisms of CRC that can lead to novel biomarkers to optimize diagnosis and/or treatment regimens are essential.

Ki67 expression has been shown to be strictly associated with cell proliferation. During interphase, the Ki67 antigen can be exclusively detected within the cell nucleus, whereas in mitosis most of the protein relocates to the chromosomal surface. Ki67 protein is present during all active phases of the cell cycle (G1, S, G2, and mitosis), but is absent from resting cells (G0). Because of these qualities, Ki67 is an excellent marker to determine the growth fraction of a given cell population, and likewise it could mark growing tumor cells [3].

Ki67 expression analysis is already used to estimate the prognosis of breast cancer and neuroendocrine tumors due to its expression pattern restricted to phases of the cell cycle in differentiating cells [4-6]. It was reported that liver metastases of CRC demonstrated a significantly higher Ki67 labeling index (Ki67-Li) [7]. Ki67 expression was found to be significantly higher in tumor tissues than in peritumoral tissues, and Ki67 levels were significantly associated with lymph node metastasis and poor prognosis of CRC [8]. However, some studies have shown that Ki67 has no correlation with patient survival $[9,10]$. We set out to investigate whether assessment of Ki67 could also help to more accurately define the prognosis for patients with CRC.

\section{KARGER}

(c) 2016 S. Karger GmbH, Freiburg 


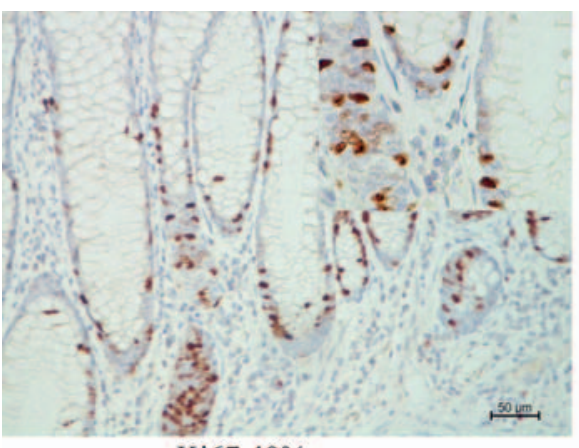

Ki67 40\%

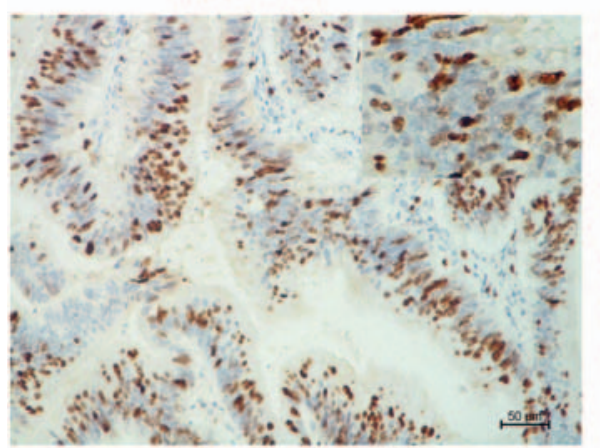

Ki67 70\%

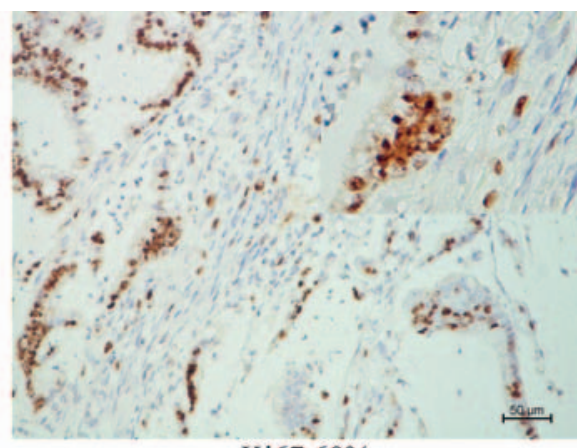

$\mathrm{Ki} 6760 \%$

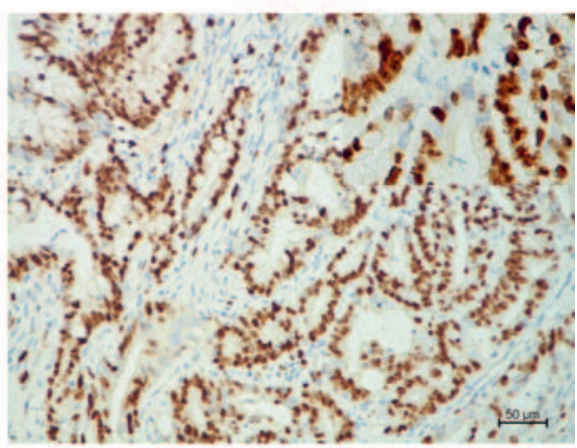

Ki67 80\%

Fig. 1. Examples of immunostaining for Ki67.

this analysis was May 2016. The median follow-up time for living patients in

\section{Patients and Methods}

\section{Patients}

The ethics committee of the Sun Yat-sen University Cancer Center approved this study, and informed consent was obtained from all patients at the beginning of the study. A total of 4,500 histologically confirmed CRC patients were recruited and 2,233 tumor tissues were obtained after operation for analysis from the Sun Yat-sen University Cancer Center between May 2011 and May 2016. All patients were of Chinese origin. The clinical and family histories of each patient were reviewed. Finally, 2,233 cases were selected for analysis following the outlined strict exclusion criteria: age less than 18 years and older than 85 years, severe complication, multiprimary cancer, synchronous and metachronous CRC, family history (first-degree and second-degree relatives with any cancer), familial adenomatous polyposis, and death not from tumor-related causes. The primary tumor site was categorized as proximal colon if the tumor was located above the splenic flexure or distal colon if it was located at or below the splenic flexure and rectum. Stage I (T1-2 N0) and stage II (T3-4 N0) CRC patients without high-risk clinical features (e.g. T4 stage, bowel perforation or clinical bowel obstruction, inadequate lymph node sampling, poorly differentiated history) were treated with radical surgery or endoscopic removal of the tumor alone. Stage II (T3-4N0) CRC patients with high-risk clinical features received chemotherapy comprising capecitabine; folinic acid +5 -fluorouracil (5-FU)+oxaliplatin (mFOLFOX); and capecitabine+oxaliplatin (XELOX). Stage III (TxN1-2) patients received radical surgery and 12 cycles of adjuvant mFOLFOX/XELOX within a 6-month period. All stage IV $(\mathrm{T} \times \mathrm{N} \times \mathrm{M} 1)$ patients with local tumor complications or resectable metastases received palliative surgery or radical surgery. The first-line treatment for stage IV CRC was the mFOLFOX/FOLFIRI (folinic acid+5-FU+irinotecan) regimen. 89 patients with rectal cancer also received neo-chemoradiotherapy. Responses were evaluated in accordance with the RECIST guidelines. After surgery, tumor recurrence was detected by physical examination, serum carcinoembryonic antigen (CEA) assay, and abdominal and thoracic imaging; patients were monitored every 3-6 months for the first 3 years, every 6 months for the following 2 years, and then annually for patients surviving beyond 5 years. The duration of follow-up was defined as the time between surgery and disease recurrence, death, or last hospital contact (scheduled follow-up or telephone contact). The cutoff date for this study was 4.3 years.

\section{Immunohistochemical Staining for Ki67}

All diagnosed CRC patients, independent of clinical criteria, were prospectively tested using IHC for the expression of MMR proteins on the second day after operation. Blocks of formalin-fixed, paraffin-embedded adenocarcinoma tissue comprising an area of normal colorectal mucosa adjacent to the tumor were selected in each case. Staining for Ki67 tissue expression was performed using the primary anti-Ki67 antibody (1:50 clone MIB-1; Dako, Tokyo, Japan). The Ki67-Li was calculated for each sample as the percentage of positively stained tumor cells among all counted tumor cells. Immunostained sections were evaluated by 2 pathologists blinded to the patients' clinical characteristics. Discordant cases were reviewed by a third pathologist to reach a consensus. Ki67 had a moderate to intense reaction in most cases, both in well-differentiated adenocarcinomas and in moderately or poorly differentiated tumors. Representative examples of immunostaining are shown in figure 1 . The mean percentage of positively stained cells for all cases was $47 \%$. In our study, a percentage of $<50 \%$ positively stained tumor cells among all counted tumor cells was considered a low Ki67-Li, and a percentage of $\geq 50 \%$ was considered a high Ki67-Li, which was in concordance with previous studies [11].

\section{Statistical Analysis}

Data are described as frequencies given in percentage values. The differences in distribution between the variables examined were assessed with the $\mathrm{X}^{2}$ or the Fisher's exact test. The primary end point used in the analysis was disease-free survival (DFS) defined as the time from the date of surgery to the first event (local or distant disease recurrence), or progression-free survival (PFS) calculated as the time from the start of surgery to clinical or radiological progression. Patients who were alive and relapse-free at the last contact were censored at the time of last follow-up. Overall survival (OS) was defined as the time elapsed from the date of surgery until tumor-induced death. Surviving patients were censored at the time of last follow-up. Median follow-up and 95\% confidence intervals (CI) were calculated using the reverse Kaplan-Meier method. The survival curve was estimated using the Kaplan-Meier method and compared using the log-rank test. Univariate and multivariate Cox proportional 
hazards models were used to explore associations between $\mathrm{Ki} 67-\mathrm{Li}$, location, age, stage, differentiation grade, and gender. The score and likelihood ratio test $\mathrm{p}$ values were used to test the statistical significance of each covariate in the univariate and multivariate Cox models, respectively. All statistical tests were two-sided, and $\mathrm{p}$ values $\leq 0.05$ were considered statistically significant. Statistical analyses were performed using SPSS software (IBM Corp., Armonk, NY, USA).

\section{Results}

Of the 2,233 CRC patients evaluated, 992 were found to have a low Ki67-Li with an overall prevalence of 44.4\%. 1,241 were found to have high Ki67 expression with a prevalence of $55.6 \%$. This selected patient population included 1,316 males and 917 females of which 32.8 and $22.8 \%$, respectively, were found to present with a high Ki67-Li while the male versus female distribution of low Ki67Li was 26.2 and $18.3 \%$, respectively. Detailed clinicopathological information for the patients included in this study is shown in table 1 . In addition, we found that a high Ki67-Li was more likely to be noticed in the right $(59.6 \%)$ or left $(60.3 \%)$ colon versus the rectum $(49.9 \%)(\mathrm{p}<0.001)$, and in older $(40-59$ years, $56.3 \%$; $60-85$ years, $56.9 \%$ ) versus younger ( $20-39$ years, $46.3 \%$; $\mathrm{p}=0.028$ ) patients. Gender, cancer stage, T (tumor) stage, N (lymph node) stage, $M$ (metastasis) stage, and pathological differentiation showed no statistical differences in univariate Cox analysis ( $p$ $>0.05$ ) for Ki67 expression. All parameters were dichotomy variables. Among the variables analyzed in the multivariate Cox model, age (hazard ratio (HR) 2.29, 95\% CI 2.23-2.33, p = 0.025) and location (HR 2.36, 95\% CI 2.31-2.38, $\mathrm{p}=0.020$ ) were significantly associated with DFS. Age (HR 2.15, 95\% CI 2.09-2.18, p = 0.022) and location (HR 2.40, 95\% CI 2.36-2.43, p = 0.018) also had a statistically significant correlation with OS.

\section{Assessment of Ki67-Li as Prognostic Marker}

Patients with stage III CRC and a high Ki67-Li had a statistically significant improvement in DFS (70\%) and OS (75\%) compared to patients with low Ki67-Li tumors (DFS 61\%; HR 0.72, 95\% CI $0.55-0.95, \mathrm{p}=0.02$ and OS 64\%; HR 0.68, 95\% CI 0.51-0.91, $\mathrm{p}=$ 0.008). We also found significant improvement in the 3-year PFS for stage IV patients in the high Ki67-Li group (PFS 14\%) versus the low Ki67-Li group (PFS 10\%) (HR 1.23, 95\% CI 1.04-1.57, p = 0.02). However, OS showed no statistical difference in stage IV patients with high Ki67-Li tumors (OS 19\%) versus low Ki67-Li tumors (OS 18\%) (HR 1.22, 95\% CI 0.99-1.51, p =0.06). From the analysis of stage I patients, we found no differences in the 3-year DFS or OS between high Ki67-Li patients versus those with a low Ki67-Li (DFS 94 vs. 93\%; HR 0.84, 95\% CI 0.35-2.03, p = 0.70 and OS 95 vs. $93 \%$; HR $0.69,95 \%$ CI $0.27-1.74, \mathrm{p}=0.43$ ). Likewise, no difference was found in the 3 -year DFS and OS of high versus low Ki67-Li stage II patients (DFS 83 vs. 78\%; HR 0.77, 95\% CI 0.57$1.05, \mathrm{p}=0.09$ and OS 85 vs. $81 \%$; HR $0.82,95 \%$ CI $0.59-1.13, \mathrm{p}=$ 0.22). The survival plots for Ki67-Li are shown in figure 2.

\section{Discussion}

Here, we evaluated the expression of Ki67 in CRC tissues and whether assessment of the Ki67-Li could be a valuable prognostic tool in this disease. We found that a high Ki67-Li appeared to be an independent and good prognostic biomarker for survival in stage III and IV CRC patients while it provided no evidence for a prognostic effect in stage I or II CRC patients. The reasons for the discrepancies between the effect of Ki67 expression in the various CRC stages are not clear. However, the differences may account for the many roles of Ki67 in cell growth and regulation.

It has been previously shown that the rate of tumor cell proliferation can be assessed by detecting the fraction of cells with nuclei expressing Ki67 [6]. The Ki67 antigen binds to the nuclei of proliferating cells throughout the cell cycle, except during the G0 and early G1 phases, marking cellular expansion [12]. Monitoring of the Ki67 protein has already been used as a clinical predictor for the prognosis of breast cancer and neuroendocrine tumors due to its expression pattern restricted to growth phases of the cell cycle found in differentiating cells $[4,5]$. Nuclear staining of Ki67 was also shown to be significantly associated with disease-specific survival in a multivariate model of clear cell renal cell carcinoma and provided the ability to predict disease outcome [13]. It has also been reported that neoadjuvant chemoradiotherapy with cetuximab decreased the levels of Ki67-Li in rectal cancer [14]. With regard to colon tissue, Ki67 was found to be highly expressed in the proliferative basal half of the crypt [15]. Also, it has been shown that Ki67 was significantly elevated in ulcerative colitis (UC) colons compared to non-UC control samples, indicating that proliferation is enhanced in UC colonic mucosa [16]. With regard to CRC itself, it has been reported that Ki67 expression correlates with patient age, pathological tumor differentiation, and lymph node involvement [17]. In addition, a strong correlation between Ki67 expression has also been seen in all levels of epithelial dysplasia and tumor grades [18]. In another study, Ki67-Li was found to significantly correlate with lymph node metastasis, but not tumor size, age, or gender, in rectal adenocarcinomas [19]. It has also been shown that Ki67 expression is not related to atypia [20].

From our large dataset of Chinese CRC patients studied here, we found that high Ki67-Li correlated with old age and better survival for stage III or IV colon cancers, but not with gender, tumor stage, or pathological differentiation. Overall, the results of our attempt to find an association between $\mathrm{Ki} 67-\mathrm{Li}$ and prognosis for the general population of patients with CRC were conflicting. Inconsistent effects of Ki67 expression with regard to patient outcomes also appear to have been evidenced in other studies. A previous study using Kaplan-Meier survival analysis showed that survival was significantly shorter for CRC patients with higher expression of Ki67 versus lower [21,22]. Yet, lower TNM and Dukes stage and higher Ki67 expression and presence of Ki67 hot spot areas in histopathological samples in rectal carcinoma were associated with better survival rates [23]. High Ki67 expression was also found to correlate well with response of chemoradiation therapy in locally advanced rectal cancers [24]. In contrast to this finding, another 
Table 1. Clinicopathological characteristics of the patients

\begin{tabular}{|c|c|c|c|}
\hline \multirow[t]{2}{*}{ Characteristic } & \multicolumn{2}{|c|}{ Ki67-Li, n (\%) } & \multirow[t]{2}{*}{$\mathrm{p}$ value } \\
\hline & $\begin{array}{l}\text { low }(<50 \%) \\
(992 / 44.4)\end{array}$ & $\begin{array}{l}\text { high }(\geq 50 \%) \\
(1,241 / 55.6)\end{array}$ & \\
\hline Sex & & & 0.496 \\
\hline Male & $584(26.2)$ & $732(32.8)$ & \\
\hline Female & 408 (18.3) & $509(22.8)$ & \\
\hline Age, years & & & 0.028 \\
\hline 20-39 & $101(4.5)$ & $87(3.9)$ & \\
\hline $40-59$ & $448(20.1)$ & $576(25.8)$ & \\
\hline $60-85$ & $443(19.8)$ & $587(25.9)$ & \\
\hline Pathology & & & 0.270 \\
\hline G1 & $38(1.7)$ & $66(3.0)$ & \\
\hline G2 & $896(40.1)$ & $1,106(49.5)$ & \\
\hline G3 & $11(0.5)$ & $7(0.3)$ & \\
\hline Mucinous & $42(1.9)$ & $53(2.4)$ & \\
\hline Signet-ring & $5(0.2)$ & $9(0.4)$ & \\
\hline Cancer stage & & & 0.165 \\
\hline I & $134(6.0)$ & $197(8.8)$ & \\
\hline IIA & $281(12.6)$ & $329(14.7)$ & \\
\hline IIB & $102(4.6)$ & $123(5.5)$ & \\
\hline IIC & $19(0.9)$ & $23(1.0)$ & \\
\hline IIIA & $21(0.9)$ & $34(1.5)$ & \\
\hline IIIB & $186(8.3)$ & $266(11.9)$ & \\
\hline IIIC & $47(2.1)$ & $45(2.0)$ & \\
\hline IVA & $124(5.6)$ & $141(6.3)$ & \\
\hline IVB & $78(3.5)$ & $83(3.7)$ & \\
\hline T stage & & & 0.197 \\
\hline $\mathrm{T} 1$ & $35(1.6)$ & $60(2.7)$ & \\
\hline $\mathrm{T} 2$ & $127(5.7)$ & $177(7.9)$ & \\
\hline T3 & $519(23.3)$ & $664(29.7)$ & \\
\hline $\mathrm{T} 4 \mathrm{a}$ & $251(11.2)$ & $277(12.4)$ & \\
\hline $\mathrm{T} 4 \mathrm{~b}$ & $59(2.6)$ & $63(2.8)$ & \\
\hline $\mathrm{N}$ stage & & & 0.143 \\
\hline N0 & $619(27.7)$ & 749 (33.6) & \\
\hline N1 & $268(12.0)$ & $344(15.4)$ & \\
\hline $\mathrm{N} 2$ & $105(4.7)$ & $147(6.6)$ & \\
\hline M stage & & & 0.411 \\
\hline M0 & $790(35.4)$ & $1,014(45.5)$ & \\
\hline M1 & $131(5.9)$ & $149(6.7)$ & \\
\hline M2 & $70(3.1)$ & $75(3.4)$ & \\
\hline Location & & & $<0.001$ \\
\hline Right colon & $211(9.4)$ & $310(13.9)$ & \\
\hline Left colon & $294(13.2)$ & $446(20.0)$ & \\
\hline Rectum & $487(21.8)$ & $485(21.7)$ & \\
\hline Metastasis & & & 0.011 \\
\hline Yes & $366(16.4)$ & $393(17.6)$ & \\
\hline No & $626(28.0)$ & $848(38.0)$ & \\
\hline Alive & & & 0.005 \\
\hline Yes & $655(29.3)$ & 889 (39.8) & \\
\hline No & $337(15.1)$ & $352(15.8)$ & \\
\hline
\end{tabular}

study showed that patients with high tumor regression actually had significantly lower Ki67 expression within their tumors than nonresponders [25]. Finally, well-differentiated adenocarcinomas from liver cancers were found to have a high number of low Ki67-Li vessels that might account for differences in tumor growth [26].

Many of these earlier studies on the effects of Ki67 expression utilized only a small number of cases in their analysis. A more re- cent study in CRC that analyzed 1,653 cases reported that high Ki67 expression is an independent prognostic marker for better survival [27]. The results of this earlier study are in slight contrast to those of the current study. In our study, we also found a high Ki67-Li to be an good independent prognostic biomarker but only for stage III or IV CRC patients. We found no prognostic effect for Ki67 indexing in stage I or II CRC patients. 

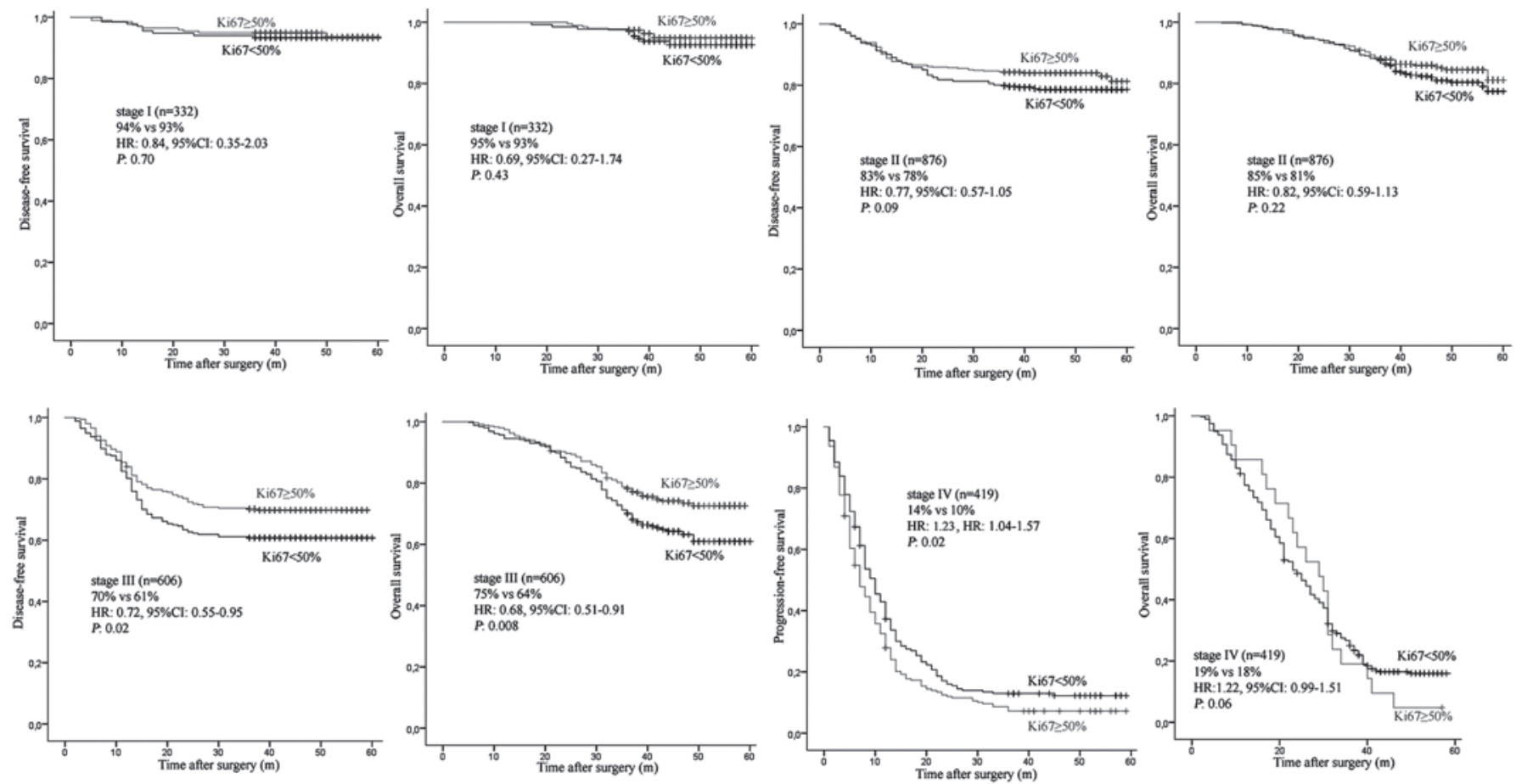

Fig. 2. Disease-free survival (DFS) and overall survival (OS) according to Ki67 expression in colorectal carcinoma (CRC) stage I-IV.

One of the criteria that might account for the above differences is the classification of Ki67 indexing. The previous study stratified the patient groups into 3 categories of low, moderate, and high whereas we used only 2 designations of either high or low. Only $17 \%$ of the previous study's 1,653 patients were considered to have a high Ki67-Li. Second, our study was conducted in only 1 hospital in China, and this population may not be comparable to the previous study's patient population nor does it necessarily reflect the entire population of China. Finally, CRC is a disease induced by multiple biological, genetic, and environmental factors that could account for differences in tumor response, and each of these elements should be examined in future studies.

\section{Conclusion}

Our data demonstrate that patients with a high Ki67-Li have a statistically significant reduction in their rates of tumor recurrence as well as better survival rates compared to patients with a low Ki67-Li for stage III CRC. While no statistically significant differences were found for patients with stage I and II tumors and a high Ki67-Li, for stage IV CRC patients Ki67-Li was an effective prognostic factor predicting PFS but not OS. Finally, we demonstrated a close correlation between high Ki67-Li and old age and colon cancer. This study provides further population-specific evidence for Ki67 expression in CRC and use of Ki67-Li monitoring for patient management.

\section{Disclosure Statement}

The authors have declared no conflicts of interest.

\section{References}

1 Torre LA, Bray F, Siegel RL, Ferlay J, Lortet-Tieulent J, Jemal A: Global cancer statistics, 2012. CA Cancer J Clin 2015;65:87-108.

2 Ferlay J, Shin HR, Bray F, Forman D, Mathers C, Parkin DM: Estimates of worldwide burden of cancer in 2008: GLOBOCAN 2008. Int J Cancer 2010;127:28932917.

3 Scholzen T, Gerdes J: The Ki-67 protein: from the known and the unknown. J Cell Physiol 2000;182: 311-322.
4 Fujimori Y, Fujimori T, Imura J, Sugai T, Yao T, Wada R, Ajioka Y, Ohkura Y: An assessment of the diagnostic criteria for sessile serrated adenoma/polyps: SSA/Ps using image processing software analysis for Ki67 immunohistochemistry. Diagn Pathol 2012;7:59.

5 Pathmanathan N, Balleine RL: Ki67 and proliferation in breast cancer. J Clin Pathol 2013;66:512-516.

6 Gerdes J, Schwab U, Lemke H, Stein H: Production of a mouse monoclonal antibody reactive with a human nuclear antigen associated with cell proliferation. Int J Cancer 1983;31:13-20.
7 Krause P, Flikweert H, Monin M, Seif AHA, Helms G, Cantanhede G, Ghadimi BM, Koenig S: Increased growth of colorectal liver metastasis following partial hepatectomy. Clin Exp Metastasis 2013;30:681-693.

8 Zhang Z, Wang M, Zhou L, Feng X, Cheng J, Yu Y, Gong Y, Zhu Y, Li C, Tian L, Huang Q: Increased HMGB1 and cleaved caspase- 3 stimulate the proliferation of tumor cells and are correlated with the poor prognosis in colorectal cancer. J Exp Clin Cancer Res 2015;34:51. 
9 Gurzu S, Szentirmay Z, Jung I: Molecular classification of colorectal cancer: a dream that can become a reality. Rom J Morphol Embryol 2013;54:241-245.

10 Debucquoy A, Goethals L, Libbrecht L, Perneel C, Geboes K, Ectors N, McBride WH, Haustermans K: Molecular and clinico-pathological markers in rectal cancer: a tissue micro-array study. Int J Colorectal Dis 2009;24:129-138.

11 Wang $\mathrm{H}$, Wen J, Wang $\mathrm{H}$, Guo Q, Shi S, Shi Q, Zhou X, Liu Q, Lu G, Wang J: Loss of expression of EphB1 protein in serous carcinoma of ovary associated with metastasis and poor survival. Int J Clin Exp Pathol 2014;7:313-321.

12 Jakob C, Liersch T, Meyer W, Becker H, Baretton GB Aust DE: Predictive value of Ki67 and p53 in locally advanced rectal cancer: correlation with thymidylate synthase and histopathological tumor regression after neoadjuvant 5-FU-based chemoradiotherapy. World J Gastroenterol 2008;14:1060-1066.

13 Weber T, Meinhardt M, Zastrow S, Wienke A, Fuesse S, Wirth MP: Immunohistochemical analysis of prognostic protein markers for primary localized clear cell renal cell carcinoma. Cancer Invest 2013;31:51-59.

14 Debucquoy A, Haustermans K, Daemen A, Aydin S, Libbrecht L, Gevaert O, De Moor B, Tejpar S, McBride WH, Penninckx F, Scalliet P, Stroh C, Vlassak S, Sempoux C, Machiels JP: Molecular response to cetuximab and efficacy of preoperative cetuximab-based chemoradiation in rectal cancer. J Clin Oncol 2009;27:27512757.
15 Dame MK, Jiang Y, Appelman HD, Copley KD, McClintock SD, Aslam MN, Attili D, Elmunzer BJ, Brenner DE, Varani J, Turgeon DK: Human colonic crypts in culture: segregation of immunochemical markers in normal versus adenoma-derived. Lab Invest 2014;94: 222-234.

16 Burum-Auensen E, Deangelis PM, Schjolberg AR, Roislien J, Andersen SN, Clausen OP: Spindle proteins Aurora a and BUB1B, but not Mad2, are aberrantly expressed in dysplastic mucosa of patients with longstanding ulcerative colitis. J Clin Pathol 2007;60:14031408.

17 Gurzu S, Jung J, Mezei T, Pavai Z: The correlation between the immunostains for p53 and Ki67 with bcl-2 expression and classical prognostic factors in colorectal carcinomas. Rom J Morphol Embryol 2007;48:9599.

18 Stromar IK, Jakic-Razumovic J: The value of immunohistochemical determination of topoisomerase IIalpha and Ki67 as markers of cell proliferation and malignant transformation in colonic mucosa. Appl Immunohistochem Mol Morphol 2014;22:524-529.

19 Jiang SM, Wang RB, Yu JM, Zhu KL, Mu DB, Xu ZF: (Correlation of VEGF and Ki67 expression with sensitivity to neoadjuvant chemoradiation in rectal adenocarcinoma). Zhonghua Zhong Liu Za Zhi 2008;30: 602-605.

20 Vernillo R, Lorenzi B, Banducci T, Minacci C, Vindigni C, Lucenti FA, Lorenzi M: Immunohistochemical expression of p53 and Ki67 in colorectal adenomas and prediction of malignancy and development of new polyps. Int J Biol Markers 2008;23:89-95.
21 Lin MX, Wen ZF, Feng ZY, He D: (Expression and significance of Bmi-1 and Ki67 in colorectal carcinoma tissues). Ai Zheng 2008;27:1321-1326.

22 Ma YL, Peng JY, Zhang P, Liu WJ, Huang L, Qin HL Immunohistochemical analysis revealed CD34 and Ki67 protein expression as significant prognostic factors in colorectal cancer. Med Oncol 2010;27:304-309.

23 Salminen E, Palmu S, Vahlberg T, Roberts PJ, Soderstrom KO: Increased proliferation activity measured by immunoreactive Ki67 is associated with survival improvement in rectal/recto sigmoid cancer. World J Gastroenterol 2005;11:3245-3249.

24 Kikuchi M, Mikami T, Sato T, Tokuyama W, Araki K, Watanabe M, Saigenji K, Okayasu I: High Ki67, Bax, and thymidylate synthase expression well correlates with response to chemoradiation therapy in locally advanced rectal cancers: proposal of a logistic model for prediction. Br J Cancer 2009; 101:116-123.

25 Jakob C, Liersch T, Meyer W, Becker H, Baretton GB, Aust DE: Predictive value of Ki67 and p53 in locally advanced rectal cancer: correlation with thymidylate synthase and histopathological tumor regression after neoadjuvant 5-FU-based chemoradiotherapy. World J Gastroenterol 2008;14:1060-1066.

26 Ceausu RA, Cimpean AM, Gaje P, Gurzu S, Jung I, Raica M: CD105/Ki67 double immunostaining expression in liver metastasis from colon carcinoma. Rom J Morphol Embryol 2011;52:613-616.

27 Melling N, Kowitz CM, Simon R, Bokemeyer C, Terracciano L, Sauter G, Izbicki JR, Marx AH: High Ki67 expression is an independent good prognostic marker in colorectal cancer. J Clin Pathol 2016;69:209-214. 